\title{
PERCEPCIÓN PÚBLICA DE LA CIENCIA: ¿QUÉ CIENCIA?; ¿QUÉ PÚBLICO? UNA APROXIMACIÓN AL IMPACTO DE LOS ENFOQUES ETNOGRÁFICOS EN LOS ESTUDIOS DE PERCEPCIÓN PÚBLICA DE LA CIENCIA'
}

\begin{abstract}
RESUMEN: El presente trabajo tiene por objeto estudiar el impacto del enfoque etnográfico en el desarrollo del campo de estudios de percepción pública de la ciencia. Con tal objeto, presento, en primer lugar, los lineamientos sobre los cuales se desarrollaron los primeros estudios y las concepciones de ciencia y público en las cuales se apoyaron. En segundo lugar, abordo las perspectivas etnográficas que dieron forma a un nuevo modelo de análisis de la percepción pública de la ciencia. Por último, a modo de cierre, propongo un breve balance de los alcances y limitaciones de este modelo de análisis y de la situación actual del campo de los estudios de percepción pública de la ciencia.

Palabras clave: Percepción Pública de la Ciencia. Modelo de Déficit. Enfoques Etnográficos.

\section{PUBLIC PERCEPTION OF SCIENCE: WHAT IS SCIENCE? WHAT IS PUBLIC? THE IMPACT OF ETHNOGRAPHIC APPROACHES IN STUDIES OF THE PUBLIC PER- CEPTION OF SCIENCE}

ABSTRACT: This paper examines the impact of the ethnographic approach in the public understanding of science. First of all, I present the guidelines on which the first studies were developed and the conceptions of science and public which supported them. Secondly, I introduce the ethnographic perspective that guided a new analysis model for the public perception of science. Finally, I conclude with a brief balance of the scope and the limitations of that perspective and the field current situation.

Keywords: Public Perception of Science. Deficit Model. Ethnographic Perspectives.
\end{abstract}




\section{INTRODUCCIÓN}

La relación del público con los desarrollos científicos y tecnológicos ha sido objeto de análisis y discusión desde el siglo XIX (IRWIN, 1995; VON GROTE; DIERKES, 2000). Sin embargo, en el siglo XX, a medida en que la ciencia se transformó en parte integral de la sociedad industrial moderna (FELT, 2000), el interés por comprender sus vínculos con la sociedad comenzó a ocupar un lugar central en las agendas académicas y políticas de diferentes países. En este contexto, surgieron los primeros documentos y estudios tendientes a analizar la relación del público con la ciencia. Hacia fines de la década de 1940 la Association of Scientific Workers (ASW) ${ }^{2}$ de Gran Bretaña editó un documento en el que se presentaban los primeros lineamientos para el estudio de la relación del público con las actividades científicas ${ }^{3}$. Allí se sostenía que una población capacitada científicamente es una mejor fuerza de trabajo para la industria, que la ciencia es un aspecto central de la cultura general de la humanidad y que una mayor comprensión de las teorías y métodos científicos es esencial por razones democráticas (IRWIN, 1995). En 1957 con el apoyo de la National Association of Scientific Writers ${ }^{4}$ (NASW) y la Rockefeller Foundation, se realizó en Estados Unidos la primera encuesta de percepción pública de la ciencia. La misma estudiaba el grado de interés y de información y la actitud hacia la ciencia y los científicos de cerca de 2000 ciudadanos estadounidenses.

Tanto el documento de la ASW como la encuesta realizada en Estados Unidos pusieron de manifiesto la preocupación de los científicos y de los diseñadores de políticas científicas por la interacción de la ciencia con el público y establecieron los parámetros en función de los cuales ésta sería estudiada en las siguientes décadas. De acuerdo con los mismos, los estudios cuantitativos realizados sistemáticamente a partir de la década de 1970 analizaron la percepción pública de la ciencia a partir de la aceptación de la asociación entre "déficit cognitivo" e indiferencia o rechazo hacia la ciencia por parte del público. Esta asociación, que dio forma al denominado "modelo de déficit", comenzó a ser fuertemente cuestionada a comienzos de la década de 1990 por nuevas perspectivas definidas como etnográficas. (IRWIN; MICHAEL 2003) ${ }^{5}$.

El presente trabajo tiene por objeto estudiar el impacto de estas perspectivas en el desarrollo del campo de estudios de percepción pública de la ciencia $^{6}$. Con tal objeto, presento en primer lugar los lineamientos sobre los cuales se desarrollaron los primeros estudios y las concepciones de ciencia y público en las cuales se apoyaron. En segundo lugar, abordo los enfoques etnográficos que dieron forma a un nuevo modelo de análisis de la percepción pública de la ciencia. Por último, a modo de cierre, propongo un breve balance de los alcances y las limitaciones, de este modelo de análisis y de la situación actual del campo. 


\section{LOS INICIOS DEL CAMPO DE LOS ESTUDIOS DE PERCEPCIÓN PÚBLICA DE LA CIENCIA}

Con el transcurso de los años, los lineamientos estipulados por el documento de la ASW y por la encuesta realizada por la NASW dieron forma a un campo de conocimiento relativamente estandarizado aunque con límites disciplinares difusos (WYNNE, 1995) y con una fuerte inscripción en el campo de la política científica (VON GROTE; DIERKES, 2000).

La encuesta de la NASW sirvió como modelo de los estudios cuantitativos tendientes a evaluar la percepción pública de la ciencia que a partir de la década de 1970 comenzaron realizarse sistemáticamente en Estados Unidos, Canadá y países de Europa como Gran Bretaña, Alemania y Francia. Las herramientas metodológicas empleadas para tal fin se centralizaban en la medición de la "alfabetización científica" (BAUER et al., 2007) definida en función de tres variables: el dominio de conceptos científicos, la comprensión de los procesos involucrados en la producción de conocimiento científico y la conciencia del impacto de la ciencia en los individuos y la sociedad (MILLER, 1983, 1992).

Por su parte el documento de la ASW encontró su continuidad en un reporte elaborado por la Royal Society de Gran Bretaña en 1985. Este escrito, titulado Public Understanding of Science, puso de manifiesto la preocupación de la esfera científica ante la creciente desconfianza hacia la ciencia por parte del público y el eventual cuestionamiento a su financiación que dicha desconfianza podía generar (IRWIN, 1995; WYNNE, 1995). En el documento se sistematizan buena parte de los lineamientos que habían operado y continuaron haciéndolo en los abordajes de los procesos de percepción pública de la ciencia en Gran Bretaña, así como en Estados Unidos y otros países de Europa.

El reporte señala que la ciencia constituye una parte integral de las sociedades contemporáneas, afectando tanto el desarrollo económico de un país como las decisiones de los individuos. En esta clave se considera que una mejor comprensión de la ciencia resulta beneficiosa en diferentes niveles. En cuanto a la economía, se señala que una ciudadanía más informada puede transformarse en una fuerza de trabajo mejor capacitada para sacar partido de los adelantos científicos y tecnológicos y así estimular la innovación a nivel de los procesos y de los productos. Asimismo, en el plano individual, se considera que un ciudadano informado tiende a tomar mejores decisiones respecto de su dieta, su salud y el medio que lo rodea, al tiempo que al comprender aspectos teóricos y metodológicos de la ciencia se apropia de parte del acerbo cultural de la humanidad. El reporte destaca también la importancia de que el público cuente con una adecuada apreciación de la naturaleza de los riesgos y de cómo interpretarlos. En suma, se considera que a mayor conocimiento, mejores decisiones individuales y sociales y mayor bienestar individual y social. De esta forma, se sostiene que la "alfabetización científica" implica que el publico está intelectualmente mejor equipado para participar en los procesos de tomas de decisiones y contribuir así con sistemas democráticos en los que el conocimiento científico se ha vuelto 
fundamental. A partir de estas consideraciones, el objetivo del reporte de la Royal Society consistió en impulsar políticas en diferentes ámbitos de la sociedad -desde la industria hasta la educación formal- tendientes a incrementar el nivel de información del público antes que a generar una evaluación crítica de las instituciones y las prácticas científicas (ROYAL SOCIETY, 1985).

Tanto el diagnóstico como las estrategias sugeridas en el documento publicado por la Royal Society y las encuestas de alcance nacional implementadas a partir de los años setenta encierran una serie de supuestos que conformaron los acuerdos básicos sobre los cuáles se desarrolló el campo de la percepción pública de la ciencia. En primer lugar, se supone que la ciencia brinda una mirada única y privilegiada del mundo y que constituye un elemento fundamental para el desarrollo de la humanidad. En segundo lugar, se asume la existencia de una cuota importante de ignorancia pública en materia de ciencia. Ignorancia o falta de comprensión que está en la base del rechazo a ciertos programas científicos por parte del público. De ello se deriva la importancia de la "alfabetización científica" para el desarrollo integral de la sociedad. Por último, se establece que la percepción es equivalente a la apreciación y al apoyo público a la ciencia. Estos lineamientos que orientaron la mayor parte de la investigación de la percepción pública de la ciencia hasta la década de 1990 se sustentaban en definiciones específicas de ciencia y de público, así como en un modelo de relación entre ambas esferas, sobre los cuales me detendré a continuación.

En el marco del reporte de la Royal Society se define a la ciencia como un sistema de investigación del mundo natural y aplicación del conocimiento derivado de esa investigación. Se supone así una continuidad entre la investigación básica y la tecnología, de acuerdo con la cual "la ciencia básica de hoy es, después de todo, la base de mucha de la tecnología del mañana" (ROYAL SOCIETY, 1985 , p. 7$)^{8}$. Este modo de entender la naturaleza y el papel de la ciencia en la sociedad, así como su articulación con la tecnología se deriva de la "imagen clásica de la ciencia”, compartida por el conjunto de los estudios metacientíficos hasta mediados de la década de $1960^{\circ}$. Desde esta perspectiva la ciencia constituye una forma de acceder a la revelación de un orden natural preestablecido antes que una práctica social condicionada por el contexto en el que se desarrolla. Así, en tanto vía de acceso privilegiada a la naturaleza, la ciencia es concebida como una empresa universal y libre de valores. Esta "imagen clásica de la ciencia” se asocia con el ideal iluminista de acuerdo con el cual el conocimiento científico constituye la principal fuerza de desarrollo de la humanidad, así como la expresión más acabada de la racionalidad humana.

La adhesión a esta imagen de la ciencia, implicó que las condiciones en las que el conocimiento científico es construido y validado quedaran fuera del foco de análisis de los procesos de percepción pública de la ciencia (WYNNE, 1995). Los abordajes se centraban, en cambio, en las prácticas comunicativas de los científicos de quienes se esperaba oficiaran de canales de comunicación del conocimiento científico hacia el público. La exclusión de la condiciones de pro- 
ducción del conocimiento científico del análisis y la limitación de la mirada de la ciencia a las habilidades comunicativas de sus practicantes implicó la consideración de la misma como un fenómeno homogéneo disciplinar y culturalmente.

El carácter homogéneo asignado a la ciencia se atribuyó también al público, el cual fue definido entonces como todo aquel "no científico", sin distinción de identidades culturales, roles o clases sociales. A este conjunto impreciso de "no científicos" o "legos", se lo consideró un mero consumidor de conocimiento con un relativo interés en mejorar su "alfabetización científica". De esta forma, la condición de "no científico" se asociaba, desde la perspectiva clásica, con la condición de receptor pasivo. Por un lado, la ciencia como emblema, de la autocrítica y la reflexión permanentes, por el otro, el público desprovisto de herramientas para aprehenderla.

La distinción en términos de aquellos que saben -los científicos- y aquellos que no -los legos- no sólo establecía un vínculo jerárquico sino también unidireccional. La información recorría un único camino posible: de los productores a los receptores (FELT, 2000). En esta clave, los enfoques tradicionales concebían al público como "mentes vacías a ser llenadas" (FAYARD, 1992) antes que cómo ciudadanos con capacidad crítica respecto al conocimiento científico. Esta concepción del público negaba la posibilidad de que éste tuviera algún tipo de saber que, aunque no científico, pudiera en algún momento ser de utilidad para la práctica o la política científica. Así, la ignorancia y la pasividad del público constituían premisas a priori de los abordajes clásicos.

Sobre estos supuestos y de acuerdo con los lineamientos antes presentados se dio forma a una agenda académica focalizada en la medición y explicación del déficit de conocimiento y a una agenda política orientada a "remediar" su existencia. De forma consiste con estas prioridades, las encuestas se transformaron en el principal instrumento metodológico para el estudio de la percepción pública de la ciencia. Focalizadas en tópicos específicos como la biotecnología (MARLIER, 1992) u orientadas a dar una visión global de la relación del público con la ciencia en una determinada región o país (BAUER et al., 1994; EVANS; DURANT, 1995) las encuestas medían la percepción pública de la ciencia mediante preguntas puntuales y estandarizadas. Asimismo, los planes tendientes a mejorar la "alfabetización científica" se transformaron en las estrategias más empleadas por los organismos públicos ${ }^{10}$.

\section{NUEVAS PERSPECTIVAS: LOS ESTUDIOS ETNOGRÁFICOS}

Hacia comienzos de la década de 1990 el enfoque clásico para el análisis de la percepción pública de la ciencia, sustentado en el "modelo de déficit" y con las técnicas cuantitativas como herramienta metodológica hegemónica, comenzó a ser fuertemente cuestionado por abordajes centrados en la relación ciencia- 
sociedad (BAUER et al., 2007). Estos enfoques de carácter etnográfico cuestionaron los supuestos ontológicos, epistemológicos y metodológicos de los abordajes precedentes. Sobre la base de diferentes tradiciones teóricas, especialmente la sociología del conocimiento científico ${ }^{11}$, autores como Brian Wynne (1992a, 1992b), Alan Irwin (1995, 2001) y Steven Epstein (1996), promovieron un nuevo modelo de análisis basado en una redefinición del objeto de estudio, así como de los modos de aprehenderlo.

Más allá de las diferencias existentes en las propuestas de estos autores, todos ellos sostienen por igual que la relación entre la ciencia y el público se desarrolla en contextos sociales específicos y que, por lo tanto, éstos deben ser parte central del análisis de los estudios de percepción pública. En estrecha conexión con esta tesis ofrecen una imagen de la ciencia que habilita el cuestionamiento de los estándares e instituciones científicas y una noción de público que rompe con la pasividad que asignaba al mismo la perspectiva clásica.

Tomando como punto de partida la imagen de la ciencia desarrollada por la sociología del conocimiento científico los enfoques etnográficos impugnaron la noción de ciencia sobre la cuál se habían desarrollado hasta el momento los estudios de percepción pública. Así, concibiendo a la ciencia como una práctica social, disciplinar y culturalmente heterogénea, enfatizan el carácter relativo del conocimiento científico (LÉVY-LEBLOND, 1992), la importancia de analizar la percepción de la "ciencia en acción" (SHAPIN, 1992; YEARLEY, 1994, 2000) y de considerar los intereses implícitos en la práctica científica (EPSTEIN, 1996).

En esta línea, los enfoques etnográficos consideran simultáneamente y de forma relacional los contenidos formales del conocimiento científico, los métodos y procesos de la ciencia y sus formas de institucionalización, organización y control. Mientras que los enfoques precedentes tendían a reconocer tan sólo las dos primeras dimensiones, la nueva perspectiva encuentra en la dimensión institucional y organizacional las claves que moldean las experiencias del público con la ciencia y las respuestas a la misma que éste genera (WYNNE, 1992b).

Esta nueva concepción se asocia con una fuerte crítica de las instituciones científicas y de su capacidad reflexiva en el marco de su relación con el público. Los enfoques etnográficos tienden a considerar esta incapacidad de la ciencia para comprender y reflexionar sobre su propia constitución social y cultural como la principal fuente de la desconfianza del público y, consecuentemente, la traba en la relación entre ambas esferas. De este modo, la mayor reflexividad de la ciencia es vista como condición necesaria para mejorar su percepción pública y reforzar su legitimidad (WYNNE, 1995).

Al igual que la ciencia, el público es comprendido por los enfoques etnográficos en términos contextuales. Ello implica considerar que toda percepción, comprensión o apropiación de la ciencia por parte del público está mediada por las diferentes identidades sociales que lo definen. Es en el marco de las identidades sociales donde el conocimiento científico es recibido, experimentado, rechazado o reformulado. Identidades sociales que son entendidas como el resultado de procesos de interacción social en los cuales se generan y modifican 
constantemente. La adscripción de los actores a múltiples identidades sociales así como su reformulación permanente, dotan a las mismas de una flexibilidad que se traslada a los procesos de percepción y apropiación de la ciencia y en la cuál los enfoques etnográficos ponen un marcado énfasis (WYNNE, 1992a).

Asimismo, en el marco de estas perspectivas de análisis se cuestiona la distinción entre científicos y legos como única forma de entender la relación entre la ciencia y el público, bajo el supuesto de que el déficit cognitivo no permite concluir que un grupo es menos capaz que otro para comprender las necesidades vinculadas con la ciencia en las sociedades modernas (VON GROTE; DIERKES, 2000). Se señala, por el contrario, que el público o bien cuenta con un conocimiento del mundo social y natural que forma parte de su entorno cotidiano que supera al de los científicos, o bien está capacitado para decidir en quien confiar. El público es considerado capaz de manejarse en un entorno científico sin conocer o comprender necesariamente todos los aspectos técnicos que éste involucra, es poseedor de conocimientos específicos que pueden ponerse en juego en los procesos de percepción pública de la ciencia (WYNNE, 1995; IRWIN; MICHAEL, 2003).

El enfoque etnográfico no sólo cuestiona el uso del déficit cognitivo como criterio de demarcación entre público y científicos sino también su consideración como una premisa a priori. Así, la ignorancia del público se transformó en un objeto de estudio en sí mismo, un fenómeno asociado con escenarios y motivaciones completamente divergentes. En esta clave los enfoques etnográficos reconocen diferentes "discursos de la ignorancia" (IRWIN; MICHAEL, 2003). Por un lado, el discurso en el que la falta de conocimiento científico como resultado de no saber se asocia a una incapacidad mental para entender la ciencia. Un segundo tipo de discurso de la ignorancia es aquel en que el no saber encuentra su justificación en la división del trabajo social. La ignorancia se inscribe en un relato en el que la diferencia entre la ciencia y otras esferas de acción es funcional y la relación que se establece entre la ciencia y el público es de complementariedad. Por último, un tercer tipo de discurso en el que el "no saber" es producto de una elección deliberada del público. Aquí el conocimiento científico es conscientemente puesto entre paréntesis, ignorado, abandonado o evitado porque es percibido como una distracción respecto de lo que se considera más importante.

$\mathrm{Al}$ reconocer que el público está capacitado para reflexionar por qué algunos hechos son considerados científicos y otros no, por qué algunas fuentes de conocimiento son fiables y otras no, por qué se elige confiar en algunos expertos y no en otros, los enfoques etnográficos consideran que éste puede involucrarse en procesos de "epistemología lega" (IRWIN; MICHAEL, 2003). Estos procesos no se focalizan en la utilización de criterios formales de validación del conocimiento científico como los procesos epistemológicos estándar. Se centran, en cambio, en la forma en que el público establece juicios sobre la verdad, la credibilidad, la utilidad, el poder, que reflejan identidades sociales o responsabilidades individuales. De esta forma, los enfoques etnográficos atribuyen al público un 
rol activo en los procesos de percepción de la ciencia al reconocerle la capacidad de apropiarse creativamente del conocimiento científico y de generar conocimiento no estandarizado, pero no por ello menos valioso en determinados contextos.

Como resultado de las nuevas formas de comprender la ciencia y el público se modificó el modo de comprender los vínculos entre ambos. Del modelo verticalista en el que la ciencia derrama su conocimiento hacia un público ignorante, se pasó a un modelo dialoguista en el que es posible pensar la retroalimentación de ambos polos de la relación. Si la clave de una exitosa relación entre la ciencia y el público era para la perspectiva clásica una fluida y eficaz comunicación de los conocimientos de la esfera científica a la esfera pública para los enfoques etnográficos la clave del vínculo es el establecimiento de lazos de confianza que garanticen la comunicación y la retroalimentación de ambos polos. Es decir, las cuestiones relacionadas con la percepción pública del la ciencia se vinculan con la confianza y la credibilidad que el público está dispuesto a asignarles a las instituciones antes que con las capacidades del público para comprender información técnica (WYNNE, 1992a). Tanto la confianza como la credibilidad son consideradas también en términos contextuales antes que como valores intrínsecos a los actores, a la información o a las instituciones. Derivan de relaciones sociales, redes e identidades y, por lo tanto, son variables contingentes que influyen en la percepción de la ciencia al tiempo que dependen de la naturaleza de esas identidades y relaciones en constante transformación.

Ciertamente este modelo de análisis requería una herramienta metodológica completamente diferente a las encuestas. Para los enfoques etnográficos éstas relevan información de escasa utilidad para una línea de análisis interesada en explorar los significados de ciencia que se ponen en juego en todo proceso de percepción pública de la ciencia (WYNNE, 1992a). Con el objeto de mostrar algo más que niveles de aprecio por la ciencia de forma abstracta y descontextualizada, se desarrollaron estudios microsociales tendientes a dar cuenta de la interacción entre la ciencia y el público en contextos específicos. Entre los más reconocidos se encuentran los estudios de Wynne (1992b) y Epstein (1996).

Wynne estudió las reacciones de los pastores de ovejas de la región de Cumbria en el norte de Inglaterra frente a las restricciones impuestas a su actividad a partir del accidente nuclear de Chernobyl en 1986. El estudio de caso le permite a este autor mostrar, por un lado, que la percepción y apropiación de la ciencia son el producto de la interacción de los conocimientos técnicos con las identidades sociales y, por el otro, que el público está capacitado para reflexionar sobre su relación con los científicos, así como sobre el estatus epistemológico de su conocimiento lego en relación con el conocimiento experto. Por su parte, Epstein estudia la configuración del movimiento social que impactó en la investigación sobre el SIDA en los Estados Unidos a mediados de la década de 1980. A diferencia de Wynne el foco no está en una comunidad geográfica que reacciona ante un determinado problema sino que se trata de un movimiento social que se estructura en torno de dicho problema. Epstein explora las complejas 
interacciones entre científicos y activistas y los esfuerzos de ambos para influir en el desarrollo de tratamientos contra el SIDA. Señala que el movimiento fue en parte posible gracias a la pre-existencia de identidades comunitarias de los grupos de gays y lesbianas de Estados Unidos. Fueron justamente estas identidades y los recursos disponibles para estos grupos los que hicieron posible su capacidad de presión sobre los expertos, el tratamiento científico de ciertos temas e incluso sobre el testeo de ciertas drogas.

Estos estudios no agotan en absoluto la cantidad de trabajos realizados desde comienzos de la década de 1990 desde la perspectiva etnográfica, pero sí encierran ciertos atributos que todos ellos comparten: el reconocimiento del rol activo del público y de la importancia de las identidades sociales en los proceso de percepción y apropiación del conocimiento científico y en la interacción con la comunidad de expertos, así como el cuestionamiento de la "imagen clásica de la ciencia". Los trabajos de Wynne y Epstein presentan también ciertas limitaciones que se reproducen en buena parte del conjunto de los estudios etnográficos: una visión idealizada del público y su relación con el conocimiento, y la dificultad para conectar el caso estudiado con perspectivas macrosociales. Asimismo, aún cuando la redefinen, reproducen en buena medida la dicotomía ciencia-público propia de la perspectiva clásica cuya utilidad es posible poner en cuestión en un contexto donde la ciencia forma parte de tal modo de la vida cotidiana de los actores que deviene un elemento central de su identidad subjetiva y comunitaria (IRWIN; MICHAEL, 2003).

\section{A MODO DE CIERRE}

Hasta aquí he repasado brevemente la trayectoria de los estudios de percepción pública de la ciencia desde sus comienzos hasta su consolidación como un campo de conocimiento multidisciplinar. Como hemos podido ver el modelo que sustentó los primeros intentos de dar cuenta de la relación del público con la ciencia fue eficazmente cuestionado a partir de la década de 1990 por los estudios etnográficos.

Su crítica a los supuestos ontológicos, epistemológicos y metodológicos del enfoque clásico posibilitó un abordaje más complejo y fecundo de la relación del público con la ciencia. La imagen de la ciencia y del público que ofrecieron obligaron a repensar los acuerdos teóricos y metodológicos en torno de los cuales se había configurado el campo. Actualmente, el "modelo de déficit" ha sido abandonado o redefinido aún por aquellos que no necesariamente adoptan perspectivas etnográficas (STURGIS; ALLUM, 2004). Asimismo, el cuestionamiento de las encuestas como herramientas metodológicas y el uso de nuevas de estrategias de investigación ampliaron los recursos metodológicos para el estudio de los procesos de percepción pública de la ciencia (IRWIN, 2001; YEARLEY, 2000). 
Sin embargo, el campo continúa presentando limitaciones tanto teóricas como metodológicas (VACCAREZZA, 2007). Por una parte, la idealización del público característica de buena parte de perspectivas etnográficas lleva en ciertos casos a una suerte de inversión del "modelo de déficit" en virtud de la cual los problemas de la relación entre científicos y público son consecuencia de la falta de conocimiento y de una actitud negativa, no ya del público, sino de las comunidades científicas (CORTASSA, 2007). En esta línea, cabe preguntarse por la viabilidad de un enfoque que pretende asignar igual peso a ambos polos de la relación ciencia-público conservando dicha dicotomía. Tal vez, la condición de posibilidad de una perspectiva efectivamente simétrica sea el abandono de la dicotomía. Por otra parte, la evaluación de los estudios cuantitativos como estrategias metodológicas inadecuadas para dar cuenta de la interacción entre ciencia y sociedad (WYNNE, 1995) supone una restricción metodológica que contribuye a la desconexión entre las perspectivas macro y microsociológicas. La complementariedad de técnicas cuantitativas y cualitativas así como una mayor conexión con la teoría macrosociológica permitirían no sólo ampliar la comprensión del los procesos de percepción pública de la ciencia, sino también de nuevas formas de producción de conocimiento, de la ciudadanía contemporánea y de las sociedades actuales en general (VON GROTE, DIERKES, 2000b; IRWIN; MICHAEL, 2003).

\section{NOTAS}

${ }^{1}$ Una versión previa de este trabajo fue presentada en VII ESOCITE - Jornadas Latino-Americanas de Estudios Sociales de las Ciencias y las Tecnologías, organizadas por la Sociedad Latinoamericana de Estudios Sociales de la Ciencia y la Tecnología, Río de Janeiro, 28 al 30 de mayo de 2008.

${ }^{2}$ La Association of Scientific Workers, denominada originalmente National Union of Scientific Workers, fue creada en 1918 en Gran Bretaña.

${ }^{3}$ El documento de la ASW encuentra antecedentes en los argumentos expuestos por J.B.S. Aldane en su libro Science in Everyday Life de 1939. Aldane, biólogo y activista político, había señalado la necesidad de que existiera un mayor conocimiento público de la ciencia como condición para el desarrollo efectivo de la democracia. A su juicio, ello implicaba un compromiso directo de los científicos con la transmisión del conocimiento científico al público (IRWIN, 1995).

${ }^{4}$ The National Association of Science Writers fue creada en 1934 por un grupo de periodistas especializados en ciencia.

5 Estos enfoques han sido definidos también como "constructivistas" (WYNNE, 1995) y "contextualistas" (STURGIS; ALLUM, 2004).

${ }^{6}$ En el marco de este trabajo empleo "Percepción pública de la ciencia" como traducción de la denominación inglesa del "Public Understanding of Science".

7 Empleo aquí la noción de "alfabetización científica" como traducción de la expresión inglesa "scientific literacy". A diferencia de la noción de "cultura científica" que incluye aspectos vinculados con la consideración instituciones, participación ciudadana, grupos de interés, la de "alfabetización científica" tiende a restringirse a la comprensión de ciertos contenidos y métodos científicos a nivel individual (POLINO et al., 2003). 
${ }^{8}$ Esta concepción tiene su anclaje en el "modelo lineal de innovación" elaborado por la teoría económica neoclásica. De acuerdo con el mismo, la innovación tecnológica es el resultado de un proceso de etapas sucesivas que se inicia con las actividades de investigación en ciencia básica y culmina con la "traducción" de los principios científicos a conocimientos útiles para la producción industrial. Este modelo supone que las capacidades tecnológicas se definen fundamentalmente en términos de conocimientos científicos.

"La imagen clásica de la ciencia también denominada "concepción heredada" (PUTNAM, 1989) suponía además de la consideración de la ciencia como una empresa unificada, universal y libre de valores una clara distinción entre contexto de justificación y contexto de descubrimiento, así como entre la teoría y su base empírica y una explicación racionalista de la elección y el cambio de teoría.

${ }^{10}$ En esta línea cabe mencionar la creación en 1985 del Committee on the Public Understanding of Science (COPUS) de Gran Bretaña, organismo diseñado para estimular el interés del público en las actividades científicas.

${ }^{11}$ La denominada sociología del conocimiento científico (Sociology of Scientific Knowledge) engloba a un conjunto de enfoques que modificaron el panorama de los estudios de la ciencia a partir de la década de 1970. Programas de investigación como el Programa Fuerte de la Escuela de Edimburgo (BARNES, 1977; BLOOR, 1998), el Programa Empírico del Relativismo (COLLINS, 1981), los estudios de laboratorio (KNORR CETINA, 1981; LATOUR; WOOLGAR, 1995) y la teoría del actor-red (LATOUR, 1992) dieron forma a un innovador modelo de análisis de la práctica científica que conectó elementos teóricos de tradiciones tan diversas como la filosofía de la ciencia historicista y la etnometodología. Más allá de sus diferencias teóricas y metodológicas, estos enfoques tienen en común una concepción de la ciencia como práctica social disciplinar y culturalmente heterogénea.

\section{REFERENCIAS BIBLIOGRÁFICAS}

BARNES, B. Interests and the Growth of Knowledge. London: Routledge and Kegan Paul, 1977. BAUER, M. ; DURANT, J.; EVANS, G. European Public Perceptions of Science. International Journal of Public Opinion Research, v. 6, n. 2, p. 163-186, 1994.

BAUER, M. W.; ALLUM, N.; MILLER, S. What can we learn from 25 years of PUS survey research? Liberating and expanding the agenda. Public Understanding of Science, v. 16, p. 79-95, 2007. BLOOR, D. Conocimiento e imaginario social. Tradução de Emmánuel Lizcano Rubén Blanco. Barcelona: Gedisa, 1998. Título original: Knowledge and Social Imagery.

COLLINS, H. M. Introduction: Stages in the Empirical Programme of Relativism. Social Studies of Science, v.11, n.1, especial: Knowledge and Controversy: Studies of Modern Natural Science, p. 3-10, 1981.

CORTASSA, C. Más allá del déficit cognitivo. Confianza y justicia epistémicas en la relación entre expertos y ciudadanos. Ponencia presentada en el $1{ }^{\circ}$ Congreso Argentino de Estudios Sociales de la Ciencia y la Tecnología, Bernal, 2007.

EPSTEIN, S. Impure Science: AIDS, Activism, and the Politics of Knowledge. Berkeley and Los Angeles, California: California University Press, 1996.

EVANS, G.; DURANT, J. The relationship between knowledge and attitudes in the public understanding of science in Britain. Public Understanding of Science, v. 4, p. 57-74, 1995.

FELT, U. Why should the public "understand" science? Some aspects of Public Understanding of Science from a historical perspective. In: DIERKES; M.; VON GROTE, C. (Eds.) Between Understanding and Trust: The Public, Science and Technology. Londres: Routledge, 2000, p.7-38.

IRWIN, A. Citizen Science: A Study of People, Expertise and Sustainable Development. Oxon: Routledge, 1995.

. Constructing the scientific citizen: Science and democracy in the biosciences. Public Understanding of Science, v.10, p. 1-18, 2001.

IRWIN, A MICHAEL, M. Science, Social Theory and Public Knowledge. Berkshire: Open University Press, 2003. FAYARD, P. Let's stop persecuting people who don't think like Galileo! Public Understanding of Science, v. 1, p. 15-16, 1992. 
KNORR CETINA, K. The Manufacture of Knowledge: an Essay on the Constructivist and Contextual Nature of Science. Oxford: Pergamon, 1981.

LATOUR, B. Ciencia en acción. España: Labor, 1992. Título original: Science in Action, How to Follow Scientists and Engineers through Society.

LATOUR, B.; WOOLGAR, S. La vida en el laboratorio. España: Alianza Editorial, 1995. Título original: Laboratory Life. The (Social) Construction of Scientific Facts.

LEVY-LEBLOND, J. M. About misunderstandings about misunderstandings. Public Understanding of Science, v. 1, p. 17-21, 1992.

MARLIER, E. Biotechnology and genetic engineering: what Europeans think about it in 1993. Brussels: INRA, 1993.

MILLER, J. D. Scientific Literacy: a Conceptual and Empirical Review. Daedalus, v. 112, n. 2, p. 2948, 1983.

. Towards a Scientific Understanding of the Public Understanding of Science and Technology. Public Understanding of Science, v. 1, p. 23-30, 1992.

POLINO, C.; FAZIO, M. E.; VACCAREZZA, L. Medir la percepción pública de la ciencia en los países iberoamericanos. Aproximación a problemas conceptuales. Revista Iberoamericana de Ciencia, Tecnologia, Sociedad e Innovación, n. 5, 2003.

PUTNAM, H. Lo que las teorías no son. In: OLIVÉ, L.; PÉREZ RANSANZ, A. R. (Eds.), Filosofía de la ciencia: teoría y observación. México: Siglo XXI, 1989, 312-329. Título original: What Theories Are Not?. In: NAGEL, E., SUPPES, P.; TARSKI, A. Logic, Methodology and Philosophy of Science: Proceedings of the 1960 International Congress. Stanford: Stanford University Press, 1962.

ROYAL SOCIETY. The Public Understanding of Science. London: The Royal Society, 1985. Disponível em: http:// royalsociety.org/document.asp?tip=1\&id=6903

SHAPIN, S. Why the public ought to understand science-in-the-making. Public Understanding of Science, n.1, p.27-30, 1992.

STURGIS, P.; ALLUM, N. Science in society: re-evaluating the deficit model of public attitudes. Public Understanding of Science, v. 13, p. 55-74, 2004.

VACCAREZZA, L. The Public Perception of Science and Technology in a Periphery. Science, Technology and Society, v. 12, p. 141-163, 2007.

VON GROTE, C.; DIERKES. M. Public Understanding of Science and Technology: State of the Art and Consequences for Future Research. In: DIERKES, M.; VON GROTE, C. (Eds.). Between Understanding and Trust: The Public, Science and Technology. Londres: Routledge, 2000, p.341-362. WYNNE, B. Public understanding of science research: New horizons or hall of mirrors? Public Understanding of Science, v. 1, p. 37-43, 1992a.

. Misunderstood misunderstanding: Social identities and public uptake of science. Public Understanding of Science, v.1, p. 281-304, 1992b.

. Public Understanding of Science. JASANOFF, S.; MARKLE, G. E; PETERSEN, J. C.; PINCH, T. (Eds.), Handbook of Science and Tecbnology Studies. Thousand Oaks, California: Sage. 1995, p. 361-388.

YEARLEY, S. Understanding science from the perspective of the sociology of scientific knowledge: An overview. Public Understanding of Science, v. 3, p. 245-258, 1994.

. Making systematic sense of public discontents with expert knowledge: two analytical approaches and a case study. Public Understanding of Science, v. 9, p. 105-122, 2000.

Data de recebimento: $26 / 11 / 08$ 